Revista de Psicología Vol. 31 (2), 2013 (ISSN 0254-9247)

\title{
Redes personales y locus de control en centros urbanos de la Argentina ${ }^{1}$
}

\author{
Pablo De Grande ${ }^{2}$ \\ Instituto de Investigación en Ciencias Sociales / Consejo Nacional \\ de Investigación en Ciencia y Tecnología
}

El presente estudio se propone analizar la relación entre la representación del locus de control y las estructuras de vínculos interpersonales en la Argentina. A partir de una muestra $(\mathrm{n}=1500)$ de hogares representativa de siete grandes centros urbanos (>200 mil habitantes) se examina la relación entre la externalidad del locus de control y diferentes aspectos de las redes personales de cada participante. Los resultados muestran que a mayor cantidad de relaciones disponibles se encuentran niveles inferiores de externalidad del locus de control, así como también menores niveles de externalidad al disponerse de lazos interpersonales fuera del barrio y con vínculos de nivel socioeducativo alto. En este sentido, se verifican asociaciones significativas entre representación de control del entorno y estructuras vinculares. Palabras clave: locus de control, redes personales, sociabilidad, autocontrol

\section{Personal networks and locus of control in large urban centers of Argentina}

This study analyzes the relationship between locus of control and interpersonal relations structures in Argentina. After a representative sample $(n=1500)$ of households in seven major urban centers ( $>200,000$ inhabitants), it examines the relationship between the externality of locus of control and different aspects of personal networks of each respondent. The results show that people having more relations experiment lower levels of externality of locus of control. Likewise, lower levels of externality are informed when personal ties outside the neighborhood are available, as well as ties high educational level. In this regard, significant associations are verified between control and personal relations structures.

Keywords: locus of control, personal networks, sociability, self-control

1 Investigación financiada por el Consejo Nacional de Investigaciones Ciéntificas y Técnicas (CONICET).

2 Licenciado en Sociología por la UBA, doctor en Ciencias Sociales y Humanidades por la Universidad de Quilmes y becario postdoctoral de CONICET en Sociología de la Infancia con sede en el IDICSO-USAL. Profesor adjunto de Sociología de las Relaciones Sociales en Escuela de Sociología y Sociología General en Relaciones Internacionales de la USAL. Contacto: Pedro Morán 2946. CP1419, Buenos Aires, Argentina; pablodg@gmail.com. 

Se presentan los resultados de una investigación que vincula representaciones de control del entorno con el marco de relaciones interpersonales en que cada persona se encuentra inserta. Diferentes investigaciones han abordado la temática de cómo el campo de relaciones interpersonales de una persona modifica sus formas de actuar y sus capacidades de afrontar problemas y necesidades personales y sociales. Tanto desde estudios del capital social (Lin, 2000; Arriagada \& Miranda, 2003; Grootaert, 1998; Bagnasco, Piselli, Pizzorno \& Trigilia, 2003; Woolcock, 2001), del apoyo social (Van der Poel, 1993; Liebler \& Sandefur, 1998; Castro, Campero \& Hernández, 1997; Maya Jariego \& de la Vega, 2004; Agneessens, Waegea \& Lievensa, 2006) y otros constructos (Lakey \& Heller, 1988; Muratori, Delfino \& Zubieta, 2013) se ha explicitado la importancia de las relaciones interpersonales en la vida cotidiana. Esta relevancia ha sido observada tanto en la obtención y acceso a recursos (Gutiérrez, 2005) como garantizando o facilitando condiciones para el bienestar psicológico personal (Barrón López de Roda \& Sánchez Moreno, 2001; Orcasita, Uribe, Castellanos \& Gutiérrez Rodríguez, 2012). Asimismo, la representación de control del entorno -observada en la forma del locus de control (Rotter, 1975; Oros, 2005; Visdómine-Lozano \& Luciano, 2006)- ha sido también considerada un elemento clave para la resolución de desafíos cotidianos. En particular, la convicción de poder afectar el funcionamiento del mundo circundante (la 'internalidad' del locus de control) ha probado ser un elemento positivo en el rendimiento educativo, el campo laboral, la prevención en la salud, entre otros.

El presente estudio indaga acerca de la relación entre ambos fenómenos: (a) la internalidad del locus de control, es decir, ¿qué tanto la convicción de poder afectar el mundo?, ¿depende de una mayor integración de la persona en un campo de relaciones estables y confiables? $\mathrm{Y}$, en caso de hacerlo, (b) tipos de relaciones por nivel de internalidad 
¿qué tipos de relaciones se asocian a mayores niveles de internalidad? ¿Los lazos barriales? ¿Lo familiar? ¿Las relaciones duraderas o las efímeras? Estas preguntas suponen una direccionalidad en la relación entre ambos elementos no libre de ambigüedades: ¡es el fatalismo lo que deja a una persona fuera de sus relaciones interpersonales? O por el contrario, ¡es la desafiliación social un factor que amplifica la impresión subjetiva de que el mundo se encuentra controlado por fuerzas que escapan al propio control o a la propia influencia? En el marco de esta investigación, si bien se reconocen mecanismos por los cuales existen vías de condicionamiento recíproco entre ambos fenómenos, se privilegia la perspectiva por la cual el locus de control, como representación subjetiva y relativamente variable, se encuentra condicionado por la red interpersonal de relaciones estables que la persona construyó a lo largo de su vida. Es conveniente en este sentido resaltar que esta posición se encuentra parcialmente fundada en el tipo de vínculo analizado (estables y cercanos), así como también en la constatación de que el locus de control mostraba variaciones significativas al cambiar las condiciones contextuales macroeconómicas (ante mejoras sostenidas del ingreso nacional tras las crisis económica de la Argentina del año 2001-2002 la externalidad promedio bajó sensiblemente en la población) (ODSA, 2007).

El concepto de locus de control (o lugar de control) identifica en qué medida una persona explica las respuestas de su contexto como consecuencia de sus propias acciones, o bien las visualiza como consecuencia de fuerzas que escapan a su influencia (azar, destino, terceros poderosos, etc.). Rotter (1954) desarrolló este concepto como parte de su teoría del aprendizaje social que fue presentado en 1966 (Rotter, 1975) haciendo operativa (a través de un test de 13 ítems) una medida para el nivel de confianza en términos generales que una persona tiene de lograr afectar su entorno. En la fase inicial del desarrollo del concepto, varios estudios se orientaron a sustentar empíricamente el supuesto que introduce una relación entre conducta y representación, mostrando que la percepción de que una situación se encuentra controlada por el azar, el destino o terceros con el poder de definirla está asociada a diferencias en la conducta (Phares, 1957; James \& Rotter, 1958; Holdel \& Rotter, 1962). 
Estudios posteriores, a su vez, confirmaron la relación entre el locus de control y un amplio abanico de fenómenos, tales como su impacto en la educación (Findley \& Cooper, 1983; Hendrics \& Montgomery, 1984; Otten, 1977), el desempeño laboral (Tseng, 1970), los consumos televisivos (Rodriguez, 2006) o las conductas y actitudes respecto a la salud (Goss \& Morosko, 1970; Kenneth, Strudler \& De Vellis, 1978; Norman, Bennet, Smith \& Murphy, 1998; Wallston, 2005).

Con relación a la investigación aplicada del locus de control, cabe destacar que, si bien son más numerosos los trabajos donde el locus de control se sitúa como una causa de otros efectos, existen también estudios que recuperan la perspectiva de cómo se estructura el locus de control, o que proveen información sobre sus correlaciones con otros condicionantes. Tales estudios analizan o incorporan controles sobre cómo la posición en términos sociales (observada desde diferencias fenotípicas, económicas y educativas) se asocia a diferencias esperables en la percepción de control del entorno de los sujetos. En el caso de Rodríguez (2006), al estudiar la relación entre locus de control y consumos televisivos, encontró que si bien existía una correlación entre la cantidad de horas que las personas miraban televisión y su ubicación respecto a su percepción de control, el nivel educativo era un factor de peso más importante que el locus de control. En este sentido, el capital educativo explicaba más claramente el locus de control que variables como género o edad, y reducía en gran medida los efectos del consumo televisivo sobre la percepción subjetiva. En el mismo sentido Palomar Lever y Valdés Trejo (2004), sobre una muestra de 900 individuos, pudieron constatar en México un mayor nivel de externalidad en los sectores de peor posición de clase, tanto en términos de ingresos como por nivel educativo de los encuestados. Otros factores que incidieron en menor medida en la alta externalidad fueron el nivel educativo de los padres, ser mujer, y encontrarse por debajo de los 36 ańos. En el mismo sentido, Brenlla y Despierre (2007) mostraron diferencias significativas en el locus de control por estrato socioeconómico para el período 2004-2006 en grandes centros urbanos de la Argentina, registrándose mayor nivel externalidad en los estratos más bajos (Brenlla \& Despierre, 2007). Los resultados 
de este estudio sugieren que el locus de control no puede ser tratado completamente como un rasgo psicológico inmanente a la personalidad de cada sujeto: en la Argentina, en el período de reactivación económica 2004-2006, los niveles de externalidad del locus de control bajaron casi un $30 \%$, teniendo un peso preponderante en esta variación el cambio en las representaciones de los estratos más bajos (ODSA, 2007).

Finalmente, Lachman y Weaver (1998) hallaron en Estados Unidos, sobre tres muestras de aproximadamente cuatro mil casos en total, resultados compatibles a estos últimos. En este sentido, los niveles de externalidad se reducían en los grupos de mayor nivel socioeconómico, relacionando luego estos elementos con mejoras en la salud y en el bienestar subjetivo; estos resultados son a la vez compatibles (en términos de relación entre nivel socioeconómico y locus de control) con los relevados por Farley, Cohen y Foster (1976) al estimar los diferenciales en el locus de control entre grupos de estudiantes de familias blancas y negras estadounidenses. Similares resultados encuentran Twenge y Campbell (2002) al examinar la relación entre nivel socioeconómico y autoestima, variable correlacionada parcialmente al locus de control.

¿En qué medida la estructura de relaciones interpersonales opera de manera análoga al nivel socioeconómico? Si bien los temas de la sociabilidad y la interacción personal no son nuevos en el campo de la teoría social —en términos, por ejemplo, de la preocupación por la anomia y la integración social en Durkheim (1967 [1897]), o por las formas de interacción como objeto de la sociología en Simmel (1898) - los registros sistemáticos y cuantificables de lazos, relaciones e interacciones en la vida cotidiana son relativamente recientes. Con especial impulso en torno a nuevas metodologías del 'análisis de redes sociales' (Scott, 1991), entendida como técnicas pero también como paradigma tendiente a revitalizar la importancia de medir, analizar y explicitar las relaciones sociales (Degenné y Forsé, 2004), los estudios de redes personales comenzaron a producir material empírico representativo de relaciones interpersonales. Desde las décadas de 1970 y 1980, es posible comenzar a reconocer un número creciente de trabajos en esta dirección. Trabajos clásicos como el de Granovetter (1973) sobre la 
heterogeneidad de las relaciones interpersonales respecto de su utilidad para conseguir empleos ('la fuerza de los vínculos débiles'), así como el trabajo de Fischer (1982) sobre la dinámica de las relaciones interpersonales en ámbitos urbanos de Estados Unidos mostraron que observar las relaciones interpersonales y derivar conocimiento a partir de ellas era deseable y posible. Asimismo, no menos importante fue la realización de encuestas de hogares a nivel nacional incluyendo relevamientos de relaciones interpersonales, como la General Social Survey en Estados Unidos (Burt, 1984), con experiencias similares en Holanda (Van der Gaag, 2005) y Francia (Ferrand, Mounier \& Degenne, 1999).

Por su parte, los estudios que relacionaron en diferentes contextos al locus de control con los vínculos interpersonales muestran resultados heterogéneos. Mari Cauce y Hannan (1992) examinaron diferentes tipos de apoyo social en adolescentes (familiar, escolar y de pares) con el fin de evaluar en qué grado estos tipos de apoyo, controlados por el locus de control, podían llegar a tener un efecto moderador (buffer) en los niveles de stress de los jóvenes estudiados. En esta indagación, los apoyos sociales utilizados no presentaron una asociación con el locus de control, si bien se detectaron interacciones (efectos ampliados al darse en simultáneo) de ambas variables en relación a su capacidad de moderar los niveles de estrés. Martínez García, por su parte (Martínez García, García Ramirez \& Maya Jariego, 2002), utilizó el locus de control, niveles de apoyo social y tipos de redes personales para investigar el bienestar de mujeres inmigrantes en Andalucía, tomando como grupo de control a un conjunto de mujeres locales. En dicho contexto, incluso tratándose de una muestra reducida $(\mathrm{n}=160)$, se aprecia una relación estadísticamente significativa entre locus de control y apoyo social suficiente (sufficiency of support) para el grupo de control (entrevistadas espańolas). En cambio, no hay correlaciones significativas entre locus de control y las variables generales que el estudio capta sobre soporte (ej. cantidad de personas en la red de soporte), ni entre apoyo social suficiente y locus de control en los demás grupos. Por último, Kukulu — también desde la perspectiva del apoyo social — obtuvo resultados referidos a la relación entre locus de control y vínculos interpersonales (Kukulu, Buldukoglu, Kulakaq 
\& Deniz Koksal, 2006). Al respecto, dicho estudio procuró establecer la dependencia de las habilidades sociales de estudiantes de enfermería para comunicarse y hacerse respetar (grado de asertividad), según sus condiciones de apoyo social, locus de control y habilidades comunicativas. En este marco, el estudio encontró una correlación significativa entre el apoyo social percibido de pares y el locus de control, donde un mayor apoyo se relacionó con menores niveles de externalidad; pero no encontró relación entre el locus de control y el apoyo familiar (Kukulu et al., 2006). Es relevante destacar que en el estudio de Kukulu et al. (2006), la representación del locus de control es tratada como un factor fijo, incluso si existen evidencias que las representaciones sobre sí mismo y sobre el funcionamiento del entorno sean sensibles a factores externos a lo largo del tiempo. De esta forma, los autores precisan:

El locus de control pareció afectar las habilidades comunicativas y el apoyo percibido de pares en forma directa pero tuvo un camino [una relación] indirecta con la asertividad a través de las habilidades comunicativas. Este vínculo entre locus de control y asertividad puede ser explicado por el hecho de que los estudiantes de enfermería con un locus de control interno tienen fe en sus habilidades para desarrollar habilidades comunicativas y obtener apoyo de sus pares (Kukulu et al., 2006, p. 36).

De este modo, Kukulu explica los vínculos por las representaciones, y no a la inversa, incluso si desde la formulación teórica del concepto de locus de control no es evidente cómo — dentro de umbrales nopatológicos - una mayor confianza en afectar el entorno vuelve a una persona marcadamente más social.

\section{Método}

\section{Participantes}

Los datos a partir de los cuales se realizó este análisis se basan en la Encuesta de la Deuda Social Argentina del año 2006. Dicha encuesta 
se realiza en un conjunto de aglomerados urbanos de la Argentina desde el año 2003 y releva condiciones socioeconómicas de los hogares y los individuos. En la edición del año 2006 cubrió siete grandes centros urbanos de la Argentina: Gran Buenos Aires, Gran Salta, Gran Resistencia, Neuquén-Plottier, Gran Córdoba, Gran Mendoza y Bahía Blanca, todos centros urbanos de más de 200000 habitantes. Asimismo, se incorporó a la misma un módulo de redes personales en el que se inspeccionaban vínculos que los participantes identificaban como importantes a la hora de requerirse ayuda, apoyo o consejos en sus situaciones difíciles de resolver de otro modo. De cada vínculo, a su vez, se relevaron características personales (tales que el sexo, la edad y el nivel educativo), así como atributos de la relación (tales que la duración, la frecuencia, el origen, entre otros).

El tamaño muestral fue de 1500 entrevistados (un adulto por hogar), utilizándose un procedimiento de muestreo estratificado polietápico. A partir de una lista inicial de ciudades a incluir en la muestra, la selección de casos se apoyó en información censal geo-referenciada para poder hacer, como primer paso, cuatro estratos de igual tamańo según criterios de nivel socioeducativo de las zonas geográficas de cada ciudad. A partir de esos estratos, se seleccionó aleatoriamente una cantidad de 'radios' asociados a cada punto muestral a utilizar. El 'radio' constituye la unidad censal de quinto nivel de desagregación (luego de país, provincia, departamento y fracción) disponible para el procesamiento de la información Censo Nacional de Población y Viviendas de 2001 (INDEC, 2001), correspondiente en promedio cada radio al área relativa a 300 hogares. Luego, dentro de cada radio se seleccionó aleatoriamente una manzana, para finalmente en el procedimiento de campo seleccionar al azar un hogar dentro de cada manzana seleccionada. En cada hogar seleccionado se realizaron dos revisitas en caso de no encontrarse adultos a encuestar dentro de las cuotas de edad y sexo establecidas para cada aglomerado, cubriendo así el total de 1500 adultos (mayores de 18 ańos) entrevistados. 


\section{Indicadores}

El nivel de externalidad del locus de control se relevó en la encuesta por medio de un test abreviado de locus de control (Salvia, Brenlla \& Rodriguez, 2004). El mismo se basa en un índice que indica el grado en el que la persona cree que influye en los 'refuerzos' que su entorno le proporciona (es decir, en qué medida el contexto en el que se encuentra inserto responde a sus acciones). Este test abreviado consta de cuatro ítems, extraídos de la escala de Rotter de locus de control, considerándose como individuos externos aquellos que reúnen entre dos y cuatro respuestas que marquen falta de percepción de control. Estos ítems fueron validados en pruebas piloto previas a la primera edición de la Encuesta de la Deuda Social Argentina, y con mayor exhaustividad en forma posterior a la edición de 2006 (Brenlla \& Vázquez, 2010). En términos generales, en la dimensión del locus de control, se obtuvo un total de $32 \%$ de los casos con locus de control externo, nivel que asciende a $39 \%$ si se considera solamente a las personas sin vínculos y disminuye a $29 \%$ si se observa a aquellos con vínculos.

El módulo de redes personales anexado al cuestionario del año 2006 tuvo por objeto ampliar la visibilidad de las manifestaciones de los lazos interpersonales en la población estudiada. Si bien en ediciones anteriores se habían realizado mediciones de indicadores de apoyo social — como dimensión relevante de la sociabilidad-, el nuevo módulo de redes personales debía permitir hacer más accesible información referida a los vínculos circundantes, fueran o no objeto de operaciones concretas de apoyo reciente. El diseño del módulo replicó en buena medida el cuestionario preparado por el equipo de Burt (1984) para la General Social Survey de 1985 en Estados Unidos. Respecto a este modelo se agregaron algunos ítems y se quitaron otros, dando más relevancia a lo barrial y restando información referida a los contenidos de las relaciones. El módulo para centros urbanos de la Argentina se apoyó, al igual que en el caso estadounidense, en un único generador de nombres ${ }^{3}$,

3 La estrategia de generador de nombres es usual en estudios de redes personales, y solicita a través de una pregunta inicial (el 'generador') una lista de nombres al encuestado, repreguntando luego características de cada una de las personas mencionadas (Campbell \& Lee, 1991). 
evitando la complejidad de campo de retomar listas de nombres producidas bajo diferentes escenarios mentales. El generador de nombres utilizado fue:

Con frecuencia, la gente recurre a amigos, familiares, compañeros de trabajo o conocidos cuando necesitan un consejo o ayuda para situaciones que sin ellos serían difíciles de resolver. Entre sus conocidos, sin incluir a quienes viven en su hogar, dígame por favor, solamente el nombre de las personas a las que recurriría en este tipo de situaciones (ODSA, 2006)

De esta forma, cada encuestado podía citar una serie de nombres de conocidos en su entorno (que no vivieran en el mismo hogar), para que sobre los primeros cinco de ellos se aplicaran 11 ítems completando información sobre los mismos.

Como resultado del relevamiento, 1518 vínculos fueron mencionados por los 1500 encuestados, de los cuales 822 manifestaron tener uno o más vínculos. Una cuarta parte de estos vínculos fueron descriptos como de origen familiar, algo más de la mitad fueron catalogados como vínculos de amistad, y los restantes se repartieron entre vecinos, parejas, compañeros de trabajo, estudio y otros. A nivel geográfico, fue llamativo que la mitad de los vínculos residía a un $\mathrm{km}$ o menos de la vivienda del encuestado. A su vez, en $95 \%$ de las relaciones los encuestados dijeron compartir charlas sobre temas personales importantes.

Los indicadores utilizados en este artículo a partir de este relevamiento son:

- Tamaño de la red personal: refiere a la cantidad total de vínculos mencionados en el generador de nombres.

- Origen del vínculo: este indicador responde a la pregunta 'cómo se conocieron', y permite distinguir entre vínculos creados en espacios institucionales (en un trabajo o en ámbitos educativos), en el barrio, a través de conocidos u otros orígenes.

- Tipo de vínculo: con relativa independencia al origen, los vínculos son al momento de relevarse clasificados por los encuestados en categorías sociales que organizan las relaciones. Las mismas 
permiten especificar lazos de tipo familiar (padres, hermanos, hijos, otros), lazos de amistad, de vecindad, compañeros de trabajo o estudios, u otros (residual).

- Distancia a la vivienda: indica la distancia en kilómetros entre la vivienda del encuestado y aquella del vínculo mencionado (menos de $1 / 2 \mathrm{~km}$, hasta $1 \mathrm{~km}$, hasta $2 \mathrm{~km}$, de 2 a $50 \mathrm{~km}$, más de $50 \mathrm{~km}$ ). Con ello se buscó establecer la capacidad de inserción geográfica en el aglomerado urbano de los encuestados, así como la identificación de sociabilidades de corte local.

- Educación de los vínculos: el nivel educativo de los vínculos opera como referencia de clase de los lazos declarados, permitiendo establecer una cuantificación de en qué medida es relevante el capital cultural de los lazos disponibles en sus efectos generales. Las categorías fueron: secundario incompleto o menos (menos de 12 años de estudios aprobados); secundario completo (12 ańos de estudios aprobados); universitario completo o incompleto (más de 12 años de estudios aprobados).

- Edad y sexo: caracterización de los vínculos en términos de edad y sexo.

- Contenido: el ítem incluido respecto al contenido de la relación (qué hacen o comparten las personas en esa relación) interrogó sobre si las personas hablaban o no de 'temas personales importantes', con el fin de controlar si el generador de nombres seleccionado incorporaba a la medición relaciones de tipo afectivo/personal, o solamente vínculos de tipo instrumental.

- Frecuencia: cada cuánto tenían contacto las personas, pudiendo responderse en términos de contactos diarios, semanales, mensuales o menos que mensuales.

- Duración: cantidad de tiempo (en años) desde que conoció a la persona mencionada. La escala ofrecida fue: menos de 1 año, 1 a 3 años; 3 a 6 años; más de 6 años. 


\section{Resultados}

Como se mencionó anteriormente, el presente análisis se orienta a identificar elementos de los vínculos interpersonales que afecten —en la población estudiada - la representación del locus de control. En este sentido, cabe señalar que el análisis de los efectos de las características de las relaciones personales se aplica sobre los sujetos que han manifestado disponer de vínculos, dada la imposibilidad de evaluar el efecto de las relaciones en aquellas personas que no enumeraron relaciones. A modo de referencia, sin embargo, puede encontrarse al pie de cada tabla el nivel de externalidad en el conjunto de personas que no mencionaron vínculos disponibles en su entorno. El análisis de resultados entre las redes personales y el locus de control se organiza en las siguientes tres secciones: según características de la red, según características de las relaciones y según características de los alters. Se presentan en primer lugar las características en el nivel de la red.

\section{Características en el nivel de la red}

La primera aproximación a la relación entre la red personal y el locus de control considera la red como un conjunto, caracterizando en este nivel la red en función de su tamaño (cantidad de vínculos). Respecto a la medida del tamańo, cabe destacar que la misma debe ser considerada en relación al carácter parcial de captación de lazos interpersonales que este estudio comparte con la mayoría de los estudios de redes personales; es decir, tamaño de la red entendida a partir del generador de nombres aquí utilizado. Por medio de este recorte sobre personas relevantes para los sujetos dentro de sus redes, el tamaño de la red captada mostró una proporción cercana a la mitad de la muestra (45\%) que no pudo enumerar a ninguna persona como recurso adecuado para compartir una situación conflictiva o pedir consejo fuera del hogar, como se observa en la Tabla 1, mientras que en el resto el promedio de vínculos fue cercano a dos por persona. Respecto al peso del tamaño de la red sobre la subjetividad, las variaciones en la cantidad de vínculos 
incidieron en la forma en que se manifestó el locus de control, habiendo mayor concentración de personas con locus de control externo entre aquellos que no declararon vínculos. Específicamente, el porcentaje de individuos en esta condición es algo más que el doble respecto a individuos que declaran tres o más vínculos (37,8\% contra 17,3\%).

\section{Tabla 1}

Porcentaje de población adulta (18 años y más) con locus de control externo según tamaño de la red personal, expresado en cantidad de vínculos por persona, y distribución para cada categoría. Conjunto de aglomerados, 2006

\begin{tabular}{clccc}
\hline & & $\begin{array}{c}\text { Locus de control } \\
\text { externo }\end{array}$ & Casos & $\begin{array}{c}\% \text { en } \\
\text { columna }\end{array}$ \\
\hline \multirow{3}{*}{ Tamaño de la } & Ninguno & $37,8^{\text {abd }}$ & 678 & 45,2 \\
red personal & Uno & $32,7^{\text {ac }}$ & 429 & 28,6 \\
& Dos & $25,4^{\text {bce }}$ & 257 & 17,2 \\
& Tres o más & $17,3^{\text {de }}$ & 135 & 9,0 \\
& Total & 32,3 & 1500 & 100,0 \\
\hline \multicolumn{2}{c}{ Total población sin vínculos } & 37,8 & 678 & 100,0 \\
\hline
\end{tabular}

Nota. a. Diferencias entre categorías 1 y 2 Sig. 0,001 (T-test); b. Diferencias entre categorías 1 y 3 Sig. 0,059 (T-test); c. Diferencias entre categorías 2 y 3 Sig. 0,000 (T-test); d. Diferencias entre categorías 1 y 4 Sig. 0,001 (T-test); e. Diferencias entre categorías 3 y 4 Sig. 0,085 (T-test). Fuente: Elaboración propia en base a datos de la Encuesta de la Deuda Social Argentina 2006.

En este sentido, en términos de cantidad de vínculos, se produce una asociación entre tamaño de la red y locus de control, donde a mayor cantidad de lazos menor nivel general de externalidad.

\section{Características en el nivel de las relaciones}

En segunda instancia, se presenta la vinculación entre el locus de control y los atributos correspondientes a las relaciones interpersonales de la red personal. Con estos atributos se hace referencia a elementos que en su menor grado de agregación no pueden ser enunciados por la red (como lo es el tamaño) ni es tampoco posible imputarlos a los sujetos que la relación relaciona (como lo es la edad). 
Los atributos en el nivel de la relación son particularmente importantes para dar cuenta del hecho de que la relación no solamente es una conexión actual con otra de persona (una posibilidad de intercambios), sino que, en tanto conexión, responde a un proceso pasado de conformación y desarrollo de esa relación. Estos procesos, en tanto tales, tienen una historia y en ella un anclaje en un tiempo y en un espacio social. A las personas se las conoce, se las contacta y luego se las continúa viendo, en lugares concretos. Los vínculos a su vez operan como fuentes de nuevos vínculos, creciendo la red personal desde sus nodos. En ambos fenómenos - la asistencia a espacios y la circulación de conocidos entre conocidos a través de recomendaciones, salidas, reuniones, visitas, favores - se juega buena parte de la sociabilidad en términos de producción y reproducción de las redes personales. Con esto no debe suponerse que los sujetos hagan sistemáticamente — si bien pueden hacerlo en circunstancias - una reflexión activa u operaciones voluntarias sobre su red personal en tanto tal. En forma usual, la red personal (el grupo de conocidos que se encuentran socialmente cerca) aparece a los sujetos como un emergente no previsto del curso de la vida. Como consecuencia - antes que como meta- de prácticamente todo proceso social (la entrada al mercado de trabajo; eventos asociados al nacimiento, muerte o enfermedad; la escolarización propia y la de los hijos; la migración; las transformaciones macroeconómicas del Estado o la región; las guerras; etc.) las redes personales se ven alteradas en su forma y contenido. Para los sujetos, este curso de transformaciones se mantiene opaco incluso al tratar de comprenderlo retrospectivamente; por qué alguien apareció en mi vida, por qué alguien se alejó, etc.

En este sentido, las relaciones intersubjetivas son la objetivación analítica de procesos concretos de socialización, y en tanto tales no ocurren ni pueden describirse por uno u otro sujeto (ni por el ego ni por el alter) sino que transcurren y se asientan en esa sustancia específica que abordamos bajo los términos "relación", "lazo", "vínculo". La relación es para los sujetos - y para su estudio - algo que posee características propias, en tanto representación dinámica del proceso continuo de interacción durable entre dos personas. Por esto, la influencia 
de eventos distantes en el pasado - como las características del barrio de la infancia, o la elección del lugar de estudios en la juventud— pueden mantenerse visibles muchas décadas después. Respecto a la información relevada, las características que serán analizadas del vínculo son: la intensidad o fuerza, el origen, el tipo de vínculo y la distancia entre las viviendas. La intensidad de los vínculos suele ser comprendida como un concepto complejo, que remite a una variedad de factores de la experiencia de la relación. Es posible tener contactos cotidianos sin por ello compartir temas personales; o compartirlos pero hacerlo en forma ocasional con personas con las que no se establecen vínculos durables; o tener vínculos durables que son tenidos en cuenta a instancias de resolver problemas solo muy raramente. Al respecto, cabe señalar que los vínculos estudiados pueden ser clasificados en su gran mayoría como vínculos 'fuertes', entendiendo a los mismos como vínculos durables, de alto grado de compromiso personal y de alta frecuencia de contacto (Tabla 2). En este sentido, 80,5\% de los vínculos fueron con personas que fueron conocidas hace más de seis ańos, mientras que menos del $2 \%$ de los vínculos tiene un pasado inferior al año.

Con relación a los niveles de externalidad con relación a la duración, surgieron diferencias significativas entre las relaciones con duración menor al año y el resto de las relaciones, lo que daría cuenta de un cambio cualitativo en las relaciones estudiadas a partir del año. Como segundo elemento para identificar la intensidad de las relaciones, puede observarse en la Tabla 2 el alto nivel de conversaciones en que se consignó hablar de temas personales importantes con la persona mencionada (96,5\% respondió afirmativamente). Esto es particularmente importante si se considera que el generador de nombres utilizado ponía énfasis en la resolución de problemas o situaciones, bajo el supuesto de que de ese modo se mencionarían a personas (si bien no necesariamente todas ellas) consideradas 'importantes' en sentido personal amplio y no solo en términos instrumentales; según este alto nivel de participación en charlas sobre temas personales, el instrumento parece haber funcionado correctamente. En tercer lugar, puede observarse respecto a la frecuencia de encuentros el alto nivel de frecuencia de contactos, habiéndose 
consignado prácticamente la mitad de los vínculos como de ocurrencia de contacto diario (45,9\%), llegando en forma acumulada al $91,6 \%$ si se incorporan también los vínculos con frecuencia de encuentro mensual (Tabla 2). Respecto a la representación subjetiva del locus de control, se evidenciaron diferencias en el nivel de externalidad en función de una externalidad algo mayor (casi 6pp) en los vínculos de frecuencia diaria.

\section{Tabla 2}

Porcentaje de población adulta (18 años y más) con locus de control externo según intensidad de la relación a partir de duración de las relaciones (en años), contenido de las relaciones y frecuencia de encuentros. Conjunto de aglomerados, 2006

\begin{tabular}{|c|c|c|c|c|}
\hline & & $\begin{array}{l}\text { Locus de control } \\
\text { externo }\end{array}$ & Casos & $\begin{array}{c}\% \text { en } \\
\text { columna }\end{array}$ \\
\hline \multirow{5}{*}{$\begin{array}{l}\text { Duración de } \\
\text { las relaciones } \\
\text { (años) }\end{array}$} & Menos de uno & $55,5^{\mathrm{abc}}$ & 23 & 1,6 \\
\hline & Uno a tres & $20,2^{\mathrm{a}}$ & 88 & 6,1 \\
\hline & Tres a seis & $22,7^{b}$ & 151 & 10,4 \\
\hline & Más de seis & $24,6^{c}$ & 1165 & 80,5 \\
\hline & $\mathrm{Ns} / \mathrm{Nr}$ & 15,8 & 20 & 1,4 \\
\hline \multirow{3}{*}{$\begin{array}{l}\text { Contenido en } \\
\text { las relaciones }\end{array}$} & $\begin{array}{l}\text { Habla temas personales } \\
\text { importantes }\end{array}$ & 24,7 & 1398 & 96,5 \\
\hline & $\begin{array}{l}\text { No habla temas per- } \\
\text { sonales importantes }\end{array}$ & 30,7 & 40 & 2,7 \\
\hline & $\mathrm{Ns} / \mathrm{Nr}$ & 0,0 & 10 & 0,8 \\
\hline \multirow{5}{*}{$\begin{array}{l}\text { Frecuencia de } \\
\text { encuentros }\end{array}$} & Diaria & $27,1^{\mathrm{d}}$ & 662 & 45,9 \\
\hline & Semanal & $21,8^{\mathrm{d}}$ & 664 & 46,0 \\
\hline & Mensual & 26,8 & 93 & 6,4 \\
\hline & Menos que mensual & 24,9 & 25 & 1,7 \\
\hline & Total & 0,25 & 1448 & $100,0 \%$ \\
\hline \multicolumn{2}{|c|}{ Total población sin vinculos } & 37,8 & 678 & 100,0 \\
\hline
\end{tabular}

Nota. a. Diferencias entre categorías 1 y 2 Sig. 0,004 (T-test); b. Diferencias entre categorías 1 y 3 Sig. 0,006 (T-test); c. Diferencias entre categorías 1 y 4 Sig. 0,008 (T-test); d. Diferencias entre categorías 1 y 2 Sig. 0,034 (T-test). Para las personas con vínculos, el cuadro presenta los valores contabilizando a cada una de ellas tantas veces como vínculos declararan. Fuente: Elab. propia en base a datos de la Encuesta de la Deuda Social Argentina 2006. 
En la Tabla 3 se presenta el origen como segunda dimensión relativa a las características en el nivel de las relaciones. Respecto al origen, cabe destacar en primer lugar - en consonancia con otros estudios de redes interpersonales (Grossetti, 2009) — el bajo grado en que la creación de relaciones durables está sujeta a encuentros casuales, en el sentido de encuentros con personas con las que no se comparta previamente un ámbito o un conocido en común. Tanto es así que si se agrupan los vínculos de las redes personales observadas por su origen, los vínculos con familiares, los originados a través de un conocido y los atribuidos a espacios sociales (educación, trabajo y 'el barrio'), queda cubierto el $95 \%$ de los vínculos. Dentro de estos tres grandes mecanismos de socialización (la familia, los espacios y por extensión de vínculos existentes) los vínculos provenientes de los espacios educativos muestran los menores niveles de externalidad de los encuestados $(11,2 \%)$, mientras que los más altos se asocian a nexos provenientes de espacios familiares y barriales $(30,6 \%$ para familiares, $32,2 \%$ para a través de otro familiar y $30,8 \%$ para conocidos en el barrio). Los contactos a través de amigos, por su parte, muestran niveles intermedios de externalidad en los 'egos', ubicándose en forma promedio en 20,3\%.

Cabe señalar también que al analizarse el origen del vínculo en relación al locus de control, es posible reconocer un contenido propio del ámbito de construcción del vínculo (familiar, barrial, escolar, etc.) que opera con independencia al mecanismo de generación del mismo (personal directo, personal mediado, institucional). Es decir, que tanto un vínculo con un familiar como un vínculo obtenido 'a través de un familiar' se asocian a un sustrato o efecto común en términos de externalidad del locus de control. Posiblemente esto pueda explicarse en el sentido en que más allá del modo de concretarse, las relaciones comparten el matiz común de dar cuenta de un 'estilo de vida', o al menos de un conjunto de vivencias y anclajes ligados a ciertos ámbitos (por ejemplo 'lo familiar') como contenido amplio, como tópico organizador de creencias, conductas y opiniones. En la relación con las representaciones del locus de control, de hecho, se muestran más relevantes estas distinciones de contenidos institucionales del vínculo (¡son del barrio 
o de un trabajo?, ¿los conocí por un amigo o por un familiar?) que el mecanismo por el que se produjo el conocimiento, es decir, si fue en forma mediada por otro vínculo (a través de...) o en el contexto de compartir un cierto ámbito. De esta forma, los vínculos capturan la dualidad de lo histórico y de lo ahistórico a la vez; la relación es hoy de amistad, pero se inició en un 'conocido de', pudiéndose reconocer en el tiempo la impronta de este pasado.

\section{Tabla 3}

Porcentaje de población adulta (18 años y más) con locus de control externo según origen del vinculo y distribución para cada categoría. Conjunto de aglomerados, 2006

\begin{tabular}{|c|c|c|c|c|}
\hline & & $\begin{array}{l}\text { Locus de control } \\
\text { externo }\end{array}$ & Casos & $\begin{array}{c}\% \text { en } \\
\text { columna }\end{array}$ \\
\hline \multirow{13}{*}{ 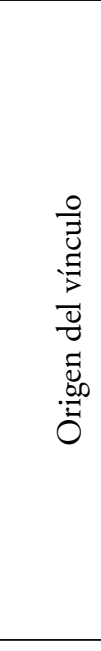 } & Espacios sociales & 22,1 & 817 & 56,4 \\
\hline & En el colegio, escuela o & $11,2^{\mathrm{a}}$ & 265 & 18,3 \\
\hline & En el trabajo & 19,3 & 139 & 9,6 \\
\hline & En el barrio & $30,8^{a}$ & 412 & 28,5 \\
\hline & A través de... & 20,0 & 207 & 14,3 \\
\hline & A través de un amigo & 20,3 & 139 & 9,6 \\
\hline & A través de una pareja & 9,0 & 22 & 1,5 \\
\hline & A través de un hijo & 8,2 & 12 & 8 \\
\hline & A través de otro familiar & 32,2 & 35 & 2,4 \\
\hline & Otros & 31,7 & 424 & 29,3 \\
\hline & Es un familiar & $30,6^{\mathrm{a}}$ & 346 & 23,9 \\
\hline & Otro & 40,1 & 71 & 4,9 \\
\hline & $\mathrm{Nr} / \mathrm{Ns}$ & 4,0 & 7 & 0,5 \\
\hline & Total & 24,6 & 1448 & $100,0 \%$ \\
\hline Total po & ación sin vínculos & 37,8 & 678 & $100,0 \%$ \\
\hline
\end{tabular}

Nota. Diferencias para categoría 'Colegio, escuela o universidad' con categorías 'Es un familiar' y 'En el barrio', Sig. 0,000 (T-test). Para las personas con vínculos, el cuadro presenta los valores contabilizando a cada una de ellas tantas veces como vínculos declararan. Fuente: Elab. propia en base a datos de la Encuesta de la Deuda Social Argentina 2006. 
En la Tabla 4, se presenta la información de relaciones y niveles de externalidad por tipo de vínculo. Al respecto, es posible constatar si se comparan las distribuciones de origen del vínculo (Tabla 3) con las de tipo de vínculo (Tabla 4), que mientras que el $28,5 \%$ de los vínculos tenían su origen 'en el barrio', al observarse por tipo de vínculo solo un $5,3 \%$ son entendidos como vecinos en el tipo de relación. En la categoría 'familia' puede verse también cómo solo parcialmente se superpone con el criterio de parentesco. Al interrogarse sobre el origen de las relaciones, 346 casos se indican como 'es familiar'; sin embargo, dentro de los vínculos 'familiares' (incluyendo 'otros familiares') la categoría reúne algunos casos adicionales (hay 353 en total, adicionándose 7 relaciones). Sobre el locus de control la información de tipos de vínculo es consistente con aquella del origen de los mismos, respecto a la relevancia de los diferentes ámbitos de construcción de vínculos en las trayectorias vitales. Esto se expresa en el sentido de una mayor percepción de control de sus condiciones por fuerzas externas, ajenas a su influencia, en aquellas personas ligadas por vínculos familiares, siendo el vínculo con los hijos la excepción dentro de ello y la categoría 'vecino' el escenario de menor nivel de representación de control por parte de los sujetos.

Es importante remarcar, por su parte, que la tipificación imputada a los vínculos (amigo, primo, etc.) resguarda un grado de discrecionalidad en su uso para los participantes de las relaciones. La laxitud en la atribución del tipo de relación sugiere la conveniencia de tomar a este indicador como un elemento variable por el que las personas resignifican sus vínculos. De esta forma, 'el barrio' puede ser mucho más que 'los vecinos' cuando se tienen muchos 'amigos' que viven en la cuadra. En este caso, el término 'vecinos', además de denotar un criterio de cohabitación residencial supone algo de negativo; ser 'solo vecinos' da cuenta de alguien que vive en las cercanías, pero que además, no puede ser clasificado como amigo. De igual forma, la inclusión en el campo de lo familiar de personas sin lazos de sangre da cuenta del sentido dado a lo familiar en la expresión 'es como de la familia'; lazos durables, confiables, seguros. Como última dimensión descriptiva del nivel de las relaciones será analizada la relación entre locus de control y la distancia 
geográfica entre las viviendas del respondente con sus vínculos. La dimensión espacial enmarca los vínculos como relaciones concretas que acontecen no solo en un tiempo y sino también en un espacio físico particular. Estas dos variables —el espacio social y el espacio físico, aunque socialmente significado — son condiciones de posibilidad para la actualización de las experiencias en que los sujetos interactúan, y en tanto condiciones son elementos capaces de 'condicionar'.

\section{Tabla 4}

Porcentaje de población adulta (18 años y más) con locus de control externo según tipo de vinculo y distribución para cada categoría. Conjunto de aglomerados, 2006

\begin{tabular}{|c|c|c|c|c|}
\hline & & $\begin{array}{l}\text { Locus de control } \\
\text { externo }\end{array}$ & Casos & $\begin{array}{c}\% \text { en } \\
\text { columna }\end{array}$ \\
\hline \multirow{14}{*}{ 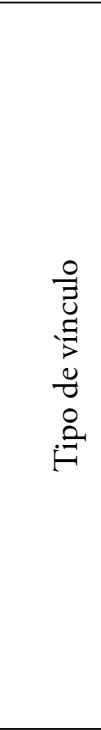 } & Familiares & 28,8 & 353 & 24,6 \\
\hline & Padre/Madre & $25,2^{\mathrm{a}}$ & 57 & 3,9 \\
\hline & Hermano/a & $32,2^{a}$ & 143 & 9,9 \\
\hline & $\mathrm{Hijo} / \mathrm{a}$ & $18,8^{\mathrm{a}}$ & 63 & 4,4 \\
\hline & Otros familiares & 32,4 & 91 & 6,3 \\
\hline & Personales no familiares & 23,4 & 1065 & 74,1 \\
\hline & Novio/a & 16,2 & 37 & 2,6 \\
\hline & Amigo & $21,1^{a}$ & 879 & 61,1 \\
\hline & $\begin{array}{l}\text { Compañero de trabajo/ } \\
\text { estudios }\end{array}$ & 22,8 & 73 & 5,1 \\
\hline & Vecino & $57,5^{\mathrm{a}}$ & 76 & 5,3 \\
\hline & Otros & 27,9 & 20 & 1,4 \\
\hline & Profesional & 32,7 & 12 & 0,9 \\
\hline & Otro & 29,8 & 8 & 0,5 \\
\hline & Total & 25 & 1438 & 100,0 \\
\hline \multicolumn{2}{|c|}{ Total población sin vínculos } & 37,8 & 678 & 100,0 \\
\hline
\end{tabular}

Nota. a. Diferencias para categorías Hermano-Hijo Sig. 0,052, Amigo-Hermano Sig. 0,012; Vecino-resto: 0,000 (T-test). Nota: Para las personas con vínculos, el cuadro presenta los valores contabilizando a cada una de ellas tantas veces como vínculos declararan. Fuente: Elab. propia en base a datos de la Encuesta de la Deuda Social Argentina 2006. 
Con relación al espacio, cabe distinguir el concepto de 'espacio relacional', de aquel de 'espacio físico', retomando a este último como un elemento pasible de ser estudiado desde una motivación sociológica. Esto es, que con independencia al espacio social de interacciones en que es posible abstraer una trama social, subyace a este el espacio físico en el que los cuerpos y objetos del primero se ubican y conforman. El espacio físico, por su parte, permite ser incorporado en el análisis —al igual que el espacio social - como un sistema de determinación de distancias, en el que es posible distinguir lo cercano de lo remoto, lo agrupado de lo disperso. La relación entre estos espacios (en última instancia, entre sus criterios de distanciamiento) es de interés para el análisis de aspectos sociales en tanto que las distancias que emite el sistema físico condicionan las interacciones en el espacio relacional. Esto se hace notar de varias maneras. Por una parte, la distancia física guarda una relación con el costo material de las interacciones. Para actualizar una relación cara a cara la copresencia es una condición, y dicha copresencia supone en relaciones entre persones físicamente distantes la necesidad de desplazarse. Para esto, el uso de medios de transporte rentados o adquiridos (trenes, micros, autos, aviones) impone erogaciones directas para costear la dinámica de los encuentros. Por otra parte, las redes vinculares y las instituciones (en su manifestación más física, sus edificios) se introducen también en estos esquemas de distancias y cercanías, provocando sistemas barriales de interacción en los que transitar de un barrio a otro, o de una región de la ciudad a otra, puede aumentar en dificultad en forma ampliada con relación a la distancia 'estrictamente física'. Esto produce formas de socialización particulares por zona (geográfica), que coordinan las distancias físicas con distancias sociales, cargando de sentido la interacción entre vecindarios, barrios, zonas y regiones.

Por último, en el marco de las relaciones, la distancia física condiciona las formas de las interacciones; antes por cartas, y ahora también por medios de comunicación a distancia (teléfono, Internet), es posible establecer interacciones en la lejanía, reproduciendo artificialmente parte de la experiencia de la interacción cara a cara. Sin embargo, además de los medios de telecomunicación, las instituciones fuera del barrio (asistir al trabajo, espacios educativos, etc.) tienen una función clave en la posibilidad 
de socializar con personas y pautas ajenas al barrio. En este sentido, estas instituciones son disruptivas del espacio local, teniendo la capacidad de producir una apertura del barrio, no sin al mismo tiempo dejar supeditada esta apertura a la continuidad de la participación en ellas.

En la Tabla 5 se observan las distribuciones y valores de locus de control en función de las distancias entre las viviendas de los vínculos y el respondente. La distribución de vínculos según localización geográfica de las viviendas se reparte en forma prácticamente homogénea entre los rangos de menos de $1 / 2 \mathrm{~km}$, de $1 / 2 \mathrm{~km}$ a $2 \mathrm{~km}$ y entre $2 \mathrm{~km}$ y $50 \mathrm{~km}$, agrupando aproximadamente un tercio de los vínculos en cada una de ellos. Fuera de estos grupos resta un 5,6\% de los vínculos que habitan fuera de la ciudad del entrevistado, en la categoría más de $50 \mathrm{~km}$. Por su parte, el carácter de físicamente localizado de los sujetos se refleja en efecto a través de sus representaciones del locus de control. La convicción de no poder afectar las condiciones del contexto decrece a medida que se dispone de contactos distribuidos fuera del barrio,

\section{Tabla 5}

Porcentaje de población adulta (18 años y más) con locus de control externo según distancia entre las viviendas y distribución para cada categoría. Conjunto de aglomerados, 2006

\begin{tabular}{llccc}
\hline & & $\begin{array}{c}\text { Locus de control } \\
\text { externo }\end{array}$ & Casos & $\begin{array}{c}\% \text { en } \\
\text { columna }\end{array}$ \\
\hline \multirow{2}{*}{ Distancia } & Menos de $1 / 2 \mathrm{~km}$ & $32,1^{\mathrm{ab}}$ & 489 & 33,7 \\
entre las & De $1 / 2 \mathrm{a} 1 \mathrm{~km}$ & $25,8^{\mathrm{ac}}$ & 230 & 15,9 \\
viviendas & De $1 \mathrm{~km} \mathrm{a} 2 \mathrm{~km}$ & 19,6 & 171 & 11,8 \\
& De $2 \mathrm{~km} \mathrm{a} 50 \mathrm{~km}$ & $17,4^{\mathrm{bcd}}$ & 463 & 32,0 \\
& Más de $50 \mathrm{~km}$ & $30,9^{\mathrm{d}}$ & 81 & 5,6 \\
& Ns/Nr & 14,4 & 15 & 1,0 \\
\cline { 2 - 5 } Total & 24,6 & 1448 & 100,0 \\
\hline Total población & 37,8 & 678 & 100,0 \\
\hline
\end{tabular}

Nota. a. Diferencias entre categorías 1 y 2 Sig. 0,099 (T-test); b. Diferencias entre categorías 1 y 4 Sig. 0,000 (T-test); c. Diferencias entre categorías 2 y 4 Sig. 0,018 (T-test); d. Diferencias entre categorías 4 y 5 Sig. 0,022 (T-test). Para las personas con vínculos, el cuadro presenta los valores contabilizando a cada una de ellas tantas veces como vínculos declararan. Fuente: Elaboración propia en base a datos de la Encuesta de la Deuda Social Argentina 2006. 
partiendo de $32,1 \%$ para contactos a menos de $1 / 2 \mathrm{~km}$ y llegando a $17,4 \%$ para aquellos que corresponden a contactos a más de $2 \mathrm{~km}$. De igual modo, cabe destacar un salto cualitativo para los contactos fuera de la ciudad (más de 50 kilómetros), donde el locus de control es similar a aquellos que poseen contactos a menos de $1 / 2 \mathrm{~km}$. Esta semejanza es asimilable a una interpretación por la cual la percepción de poder actuar sobre el propio destino está al menos parcialmente ligada a haber podido integrar la propia red en el aglomerado urbano de residencia, revirtiéndose esta condición en quienes mencionaron a personas fuera de la ciudad o a personas del vecindario inmediato.

\section{Características en el nivel de los alters}

Por último, cabe observar un conjunto atributos que refieren a características de los alters, es decir, a características de las personas que fueron señaladas como miembros de las redes personales de los sujetos. Estos atributos, a diferencia de los atributos del nivel anterior, no son determinados por la relación en tanto proceso implicado en conocerse, compartir y reconocerse. Los atributos de los alters dan pie al análisis del 'quién con quién' de las relaciones, del cual será explorada en esta sección su relación con el locus de control. La relación con otras personas - tanto la generación de nuevos vínculos como su persistencia en el tiempo — responde en mayor o menor medida a criterios o reglas de selección que operan sobre tales fenómenos. La idea de que existen estos criterios o reglas no supone necesariamente —aunque sucede en un número de aspectos- que los sujetos en forma voluntaria apliquen tales criterios al definir el grado de entusiasmo con que saludan a un antiguo conocido por la calle, o en la decisión de llamar a alguien por teléfono o no hacerlo. En este sentido, se establece la necesidad conceptual de un equilibrio en el cual las personas ni se encuentren en el extremo racionalista de construir la realidad relacional en forma planificada y ajustada a criterios explícitos, ni — en el otro extremo - reciban una cantidad de vínculos dados sin más como producto de sus interacciones sociales que no guardarían relación con sus capacidades de reflexión y acción. 
Cabe a este respecto señalar la capacidad estratégica limitada de los actores, que al mismo tiempo que ponen en juego su voluntad para continuar y nutrir sus relaciones con otras personas, se encuentran restringidos en tal ejercicio por el campo de vínculos posibles en su entorno y por los recursos de que disponen para lograrlos y sostenerlos.

De igual modo, también cabe señalar que son limitadas las capacidades de los sujetos de disimular sus preferencias e inclinaciones, es decir, que la acción estratégica en términos de vínculos se encuentra también acotada por el hecho de que los sujetos poseen un cierto nivel de 'transparencia involuntaria' por la cual sus emociones se traducen, entre otras cosas, en gestos, movimientos y formas de hablar. Por esto, parte de los matices que los sujetos aplican en sus relaciones pueden no ser conscientes y sin embargo expresar en forma consistente su escala de valores y preferencias. En las relaciones cara a cara, por ejemplo, el tono de voz y el registro gestual exponen buena parte de los pareceres de los sujetos con independencia a si ellos desean o no hacerlos visibles. Dentro de este contexto, es posible suponer que relacionarse con ciertas personas pueda estar relacionado a determinado tipo de efecto subjetivo o material, sin por ello suponer que quienes socializan lo hacen teniendo esto en cuenta para atesorar así beneficios diferenciales por tales efectos en virtud de sus redes personales.

En la Tabla 6 puede observarse que la distribución de personas con locus de control externo varía significativamente si se observa la misma según franjas etarias de los alters.

El grupo de personas con quienes relacionarse se asocia en promedio a niveles altos de externalidad son los adultos de entre 36 y 55 ańos, llegando al 29\%. La categoría donde se manifiesta la menor externalidad es en los vínculos con jóvenes de 18 a 35, cuyo nivel es de $21 \%$, estando en un nivel intermedio del $27 \%$ las relaciones con personas de 56 años y más. En la distribución de la externalidad según sexo de los alters, también se encuentran diferencias significativas, variando la misma desde 21,7\% para las relaciones con alters varones a $27,0 \%$ para las relaciones con alters mujeres. 


\section{Tabla 6}

Porcentaje de población adulta (18 años y más) con locus de control externo según edad y sexo de los alters. Conjunto de aglomerados, 2006

\begin{tabular}{llccc}
\hline & & $\begin{array}{c}\text { Locus de control } \\
\text { externo }\end{array}$ & Casos & $\begin{array}{c}\% \text { en } \\
\text { columna }\end{array}$ \\
\hline \multirow{3}{*}{ Edad del alter } & 18 a 35 & $21,0^{\mathrm{a}}$ & 643 & 45,0 \\
& 36 a 55 & $29,0^{\mathrm{a}}$ & 478 & 33,4 \\
& 56 y más & 27,0 & 309 & 21,6 \\
\cline { 2 - 5 } Sexo del alter & Total & 24,6 & 1448 & $100,0 \%$ \\
\hline \multirow{2}{*}{ Total población sin vínculos } & Varón & $21,7^{\mathrm{b}}$ & 660 & $45,6 \%$ \\
& Mujer & $27,0^{\mathrm{b}}$ & 788 & $54,4 \%$ \\
\cline { 2 - 5 } & Total & 24,6 & 1448 & $100,0 \%$ \\
\hline
\end{tabular}

Nota: a. Diferencias variable 'Edad del alter' entre categorías 1 y 2 Sig. 0,004 (T-test). b. Diferencias entre categorías 1 y 2 variables 'Sexo del alter' Sig. 0,030 (T-test). Para las personas con vínculos, el cuadro presenta los valores contabilizando a cada una de ellas tantas veces como vínculos declararan. Fuente: Elab. propia en base a datos de la Encuesta de la Deuda Social Argentina 2006.

Las particularidades que se manifiestan según los atributos de los alters dejan entrever el entramado de relaciones que existen entre las dimensiones analizadas, siguiéndose que así como establecer lazos fuera del barrio reducía las chances de tener una percepción de externalidad sobre el devenir del entorno, también se muestra en igual dirección la acumulación de lazos con varones y con personas jóvenes. En este sentido, sobre el lugar de control influyen tanto atributos que son intrínsecos a los individuos con los que las personas se relacionan, como elementos que solo existen en la relación, conformando y reforzando ambos - los rasgos de la persona, como su sexo o edad, y los rasgos de la relación, como vivir cerca o tener un lazo de amistad- las ideas de mundo y de sí mismo que cada uno se construye. En este escenario, como atributo de los alters, cabe caracterizar la posición social de los miembros de la red personal, en términos de posición alcanzada en la estratificación por capital educativo formal. ¿Cuán diferenciadamente 
influye la relación con sujetos de diferente capital educativo? Al igual que en las demás dimensiones, el capital educativo de los miembros de la red —y más ampliamente el capital cultural— no emerge, claro está, como un activo aislado y sin contexto. La disponibilidad de un nivel educativo constituye un indicador de experiencias, procesos, recursos y relaciones en los que la persona ha estado inmerso y a partir de los cuales ha podido construir su identidad y sus medios de subsistencia. Esta advertencia es importante para evitar suponer que es la educación por sí misma el elemento diferenciador que explica desplazamientos simbólicos y sociales entre personas y grupos. Por el contrario, la acreditación de un título o un reconocimiento académico — si bien es un elemento que facilita ciertos procesos, tales que una búsqueda laboral, o el acceso y uso de información técnicamente compleja - opera principalmente para la investigación como aquello que 'señala', que identifica, que da cuenta, de pasados colectivos que difieren en una multiplicidad de factores extra educativos. Por esta razones, las variaciones observadas según nivel educativo en el locus de control son interpretadas no tanto como un impacto de la educación formal en sí misma sobre las representaciones subjetivas, sino como una efectividad del nivel educativo para dar cuenta de trayectorias de socialización disímiles en función de la mayor o menor permanencia en ámbitos educativos, con las implicancias directas pero sobretodo indirectas que ellas acarrean.

Por último, en consonancia con las asociaciones mencionadas en los antecedentes entre nivel educativo y locus de control, el grado de externalidad en los encuestados varía sensiblemente si se evalúa en función del nivel educativo de los alters. En la Tabla 7 puede observarse que mientras la externalidad del locus de control llega a 36,9\% entre las personas con alters sin educación secundaria completa, este nivel baja al $15,2 \%$ si se considera las relaciones con personas con educación universitaria, ya sea completa o incompleta. Esta amplitud de distancia en las proporciones de personas con locus de control externo solo se observaban en las tablas precedentes al distinguirse entre lazos provenientes del barrio y provenientes de espacios formales educativos, pudiendo suponerse que existe una relación por cierto esperable entre 
ambas dimensiones (lugar de creación del vínculo y características del alter), en términos de mayor externalidad y menor capital educativo entre los vínculos del barrio.

\section{Tabla 7}

Porcentaje de población adulta (18 años y más) con locus de control externo según nivel educativo de los alters. Conjunto de aglomerados, 2006

\begin{tabular}{llccc}
\hline & & $\begin{array}{c}\text { Locus de control } \\
\text { externo }\end{array}$ & Casos & $\begin{array}{c}\% \text { en } \\
\text { columna }\end{array}$ \\
\hline \multirow{2}{*}{$\begin{array}{l}\text { Nivel } \\
\text { educativo } \\
\text { del alter }\end{array}$} & $\begin{array}{l}\text { Secundario incompleto o } \\
\text { menos }\end{array}$ & $36,9^{\mathrm{a}}$ & 551 & 38.1 \\
& $\begin{array}{l}\text { Secundario completo } \\
\text { incompleto }\end{array}$ & $19,9^{\mathrm{a}}$ & 420 & 29 \\
\cline { 2 - 5 } & Total & $15,2^{\mathrm{a}}$ & 476 & 32.9 \\
\hline Total población sin vínculos & 24,6 & 1448 & 100,0 \\
\hline
\end{tabular}

Nota. a. Diferencias variable 'Nivel educativo del alter' entre categorías 1 y 2 y categorías 1 y 3 Sig. 0,000; entre categorías 2 y 3 Sig. 0,079 (T-test). Para las personas con vínculos, el cuadro presenta los valores contabilizando a cada una de ellas tantas veces como vínculos declararan. Fuente: Elaboración propia en base a datos de la Encuesta de la Deuda Social Argentina 2006.

\section{Conclusiones}

Las observaciones realizadas en el análisis empírico parecen ser compatibles con la hipótesis inicial de que existe una relación entre locus de control y contexto relacional, pudiéndose identificar algunos matices al interior de las relaciones relevadas. En primer lugar, se ha podido constatar que la cantidad de vínculos con los que las personas manifestaron tener relación tuvo un importante correlato con la forma de evaluar su capacidad de afectar en el entorno, siendo más del doble la proporción de personas con locus de control externo entre quienes no tenían vínculos que entre quienes declararon tres o más vínculos. Este hecho sitúa a la interacción interpersonal como un factor privilegiado a través del cual intentar comprender fenómenos subjetivos de manera 
sistemática y significativa. En este sentido, si bien es aceptado que los individuos se encuentran insertos en un tejido social de instituciones y personas que los atraviesan simbólicamente en forma cotidiana, no es evidente que la sola medida de un indicador de cantidad de personas en su entorno pueda distinguir en una formación mental como el locus de control entre niveles que se duplican entre los grupos tal como ocurrió con el tamaño de la red.

En igual forma, hubo diferencias entre quienes tenían vínculos. La percepción de poder afectar el entorno aumentó sensiblemente al mantenerse lazos originados en espacios educativos, mientras que quienes tenían vínculos eminentemente familiares vieron reducida su percepción de control sobre el mundo circundante. Geográficamente también aparecieron tendencias diferenciadas en la manifestación del lugar de control, siendo casi el doble la proporción de personas con creencias externas entre quienes nombraron vínculos que vivían a menos de $1 / 2$ $\mathrm{km}$ en comparación a quienes contaban con relaciones dentro de la ciudad pero a más de $2 \mathrm{~km}$. Por último, el capital educativo de los alters mostró también ser un elemento altamente vinculado a la representación de control, duplicándose los niveles de externalidad según los vínculos carecieran de estudios secundarios y tuvieran estudios universitarios de algún tipo.

Retomando la hipótesis inicial planteada se señalaron varias dimensiones de la sociabilidad con comportamientos particulares respecto de la externalidad o internalidad del locus de control. Asimismo, es necesario recalcar la validez limitada de la medición del espacio relacional interpersonal (como muestra parcial de la red total de relaciones de cada persona), dando cuenta en este estudio de un tipo particular de relaciones abordado por medio del recorte de las relaciones durables, frecuentes y personales de los ámbitos urbanos investigados. 


\section{Referencias}

Agneessens, F., Waegea, H. \& Lievensa, J. (2006). Diversity in social support by role relations: A typology. Social Networks, 28(4), 427-441.

Arriagada, I. \& Miranda, F. (2003). Capital social de los y las jóvenes. propuestas para programas y proyectos. En CEPAL, División de Desarrollo Social, Volúmenes I y II, Santiago de Chile.

Bagnasco, A., Piselli, F., Pizzorno, A. \& Trigilia, C. (2003). El capital social. Instrucciones de uso. Buenos Aires: Fondo de Cultural Económico.

Barrón López de Roda, A. \& Sánchez Moreno, E. (2001). Estructura social, apoyo social y salud mental. Psicothema, 13 (1), 17-23.

Brenlla, M. \& Despierre, M. (2007). Condiciones psicológicas y autonomía de agencia. VIII Congreso Nacional de Ciencia Politica de la Sociedad Argentina de Análisis Politico. Buenos Aires, 6 al 9 de noviembre.

Brenlla, M. \& Vázquez, N. (2010). Análisis Psicométrico de la adaptación argentina de la escala de Locus de Control de Rotter. Documento de Trabajo Nro 2, Observatorio de la Deuda Social Argentina.

Burt, R. (1984). Network Items and the General Social Survey. Social Networks, 6, 293-339.

Campbell K. \& Lee B. (1991). Name generators in surveys of personal networks. Social Networks, (13), 203-221.

Castro, R., Campero, L. \& Hernández, B. (1997). La investigación sobre apoyo social en salud: situación actual y nuevos desafíos. Rev. Saúde Pública, 31 (4), 425-35.

Degenné, A. \& Forsé, M. (2004). Introducing Social Networks. Londres: SAGE Publications.

Durkheim, E. (1967 [1897]). Fragmento de El suicidio, Libro II, Capítulo V, Secciones ii y iii. Editado como artículo bajo el nombre Anomia. Relaciones humanas, Conceptos y casos. Buenos Aires: Bibliográfica Omeba. 
Farley, F., Cohen, A. \& Foster, A. (1976). Predicting locus of control in black and white college Students. Journal of Black Studies, 6(3), 299-304.

Ferrand, A., Mounier, L. \& Degenne, A. (1999). The Diversity of Personal Networks in France: Social Stratification and Relational Structures. En Networks in the Global Village. Life in Contemporary Communities, 185-224.

Findley, M. \& Cooper, H. (1983). Locus of control and academic achievement. A literature review. Journal of Personality and Social Psychology, 9, 13-16.

Fischer, S. (1982). To Dwell Among Friends. Chicago: University of Chicago Press.

Goss, A. \& Morosko, T. (1970). Relation between a dimension of internal-external control and the MMPI with an alcoholic population. Journal of Consulting and Clinical Psychology, 34(2), 189-192.

Granovetter, M. S. (1973). The strength of weak ties. American Journal of Sociology, 78, 1360-1380.

Grootaert, C. (1998). "Social Capital: The Missing Link?", Social Capital Initiative, Working Paper Series, 3, Washington DC, The World Bank Social.

Grossetti, M. (2009). ¿Qué es una relación social? Un conjunto de mediaciones diádicas. REDES, 6(2).

Gutiérrez, A. (2005). Pobre' como siempre. Córdoba: Ferreyra Editores Hendrics, L \& Montgomery, T. (1984). Educational achievement and locus of control among black adolescent fathers. Journal of Negro Research, 53, 182-188.

Holdel, K. \& Rotter, J. (1962). A nonverbal measure of extinction in skill and chance situations. Journal of Experimental Psychology, 63, 519-520.

INDEC (2001). Censo Nacional de Población, Hogares y Viviendas 2001 (CD-ROM). 
James, W. \& Rotter, J. (1958). Partial and 100\% reinforcement under chance and skill conditions. Journal of Experimental Psychology, 55, 397-403.

Kenneth, A., Strudler, B. \& De Vellis, R. (1978). Development of the multidimensional health locus of control scales. Health Education \& Behavior, 6(1), 160-170.

Kukulu, K., Buldukoglu, K., Kulakaq, O. \& Deniz Koksal, C. (2006). The Effects of Locus of Control, Communication Skills and Social Support on Assertiveness in Female Nursing Students. Social behavior and personality, 34(1), 27-40.

Lachman, M. \& Weaver, S. (1998). The Sense of Control as a Moderator of Social Class Differences in Health and Well-Being. Journal of Personality and Social Psychology, 74(3), 763-773.

Lakey, B. \& Heller, K. (1988). Social Support from a Friend, Perceived Support, and Social Problem Solving. American Journal of Community Psychology, 16(6), 811-824.

Lieber, C. \& Sandefur, G. (1998). Exchanging Social Support with Friends, Neighbors, and Coworkers. CDE Working Paper, 98-19, 1-38.

Lin, N. (2000). Social Capital: A Theory of Structure and Action. Cambridge, Reino Unido: Cambridge University Press.

Mari Cauce, A. \& Hannan, K. (1992). Life Stress, Social Support, and Locus of Control During Early Adolescence: Interactive Effects. American Journal of Community Psychology, 20(6), 787-798.

Martínez García, M., García Ramirez, M. \& Maya Jariego, I. (2002). Social support and locus of control as predictors of psychological well-being in Moroccan and Peruvian immigrant women in Spain. International Journal of Intercultural Relations, 26, 287310.

Maya Jariego, I. \& de la Vega, L. (2004). Niveles de multiplicidad y tipos de proveedores de apoyo: las redes personales de los inmigrantes indios en Argentina. IV Mesa Hispana para el análisis de redes sociales. XXIV International Sunbelt Social Network Conference, Portorož, Slovenia, mayo 12-16, 2004. 
Muratori M., Delfino G. \& Zubieta, E. (2013). Percepción de anomia, confianza y bienestar: la mirada desde la psicología social. Revista de Psicología, 31(1), 129-150.

Norman, P., Bennet, P., Smith, C. \& Murphy, S. (1998). Health Locus of Control and Health Behaviour. Journal of Health Psychology, 3(2), 171-180.

ODSA (2006). Cuestionario de la Encuesta de la Deuda Social Argentina. Recuperado el 15 de 12 de 2006, de Observatorio de la Deuda Social: http://www.uca.edu.ar/esp/sec-investigacion/esp/ subs-observatorio/page.php?subsec=cuestionarios

ODSA (2007). Barómetro de la Deuda Social Argentina / 3. Progresos Sociales 2004-2006. Buenos Aires: Fundación Arcor y EDUCA. Orcasita, L., Uribe, A., Castellanos, L. \& Gutiérrez Rodríguez, M. (2012). Apoyo social y conductas sexuales de riesgo en adolescentes del municipio de Lebrija-Santander. Revista de Psicología, 30(2), 372-406.

Oros, L. (2005). Locus de control: Evolución de su concepto y operacionalización. Revista de Psicología de la Universidad de Chile, $X I V(1), 89-98$.

Otten, M. (1977). Inventory and expressive measures of locus control and academic performance: a 5-year outcome study. Journal of Personality Assessment, 41, 644-649.

Palomar Lever, J. \& Valdés Trejo, L. (2004). Pobreza y locus de control. Revista Interamericana de Piscología, 38(2), 225-240.

Phares, E. (1957). Expectancy changes in skill and chance situations. Journal of Abnormal and Social Psychology, 54, 339-342.

Rodriguez, F. (2006). Televisión y locus de control: cultivo del miedo y el autoritarismo en los televidentes norteamericanos. XII Encuentro Latinoamericano de Facultades de Comunicación Social, Bogotá, Colombia, 25 al 28 de septiembre.

Rotter, J. B. (1954). General Principles for a Social Learning Framework of Personality Study. En J.B. Rotters, Social Learning and clinical psychology (pp. 82-104). Englewood Cliffs: Prentice-Hall Inc. 
Rotter, J. B. (1975). Some problems and misconceptions related to the construct of internal versus external control of reinforcement. Journal of Consulting and Clinical Psychology, 43(1), 56-67.

Salvia, A., Brenlla, M. \& Rodriguez, M. (2004). Competencias psicosociales. En ODSA Barómetro de la Deuda Social Argentina (pp. 155-170). Buenos Aires: EDUCA.

Scott, J. (1991). Social Network Analysis. A Handbook. Londres: Sage Publications.

Simmel, G. (1898). The persistence of social groups. The American Journal of Sociology, 3(5), 662-698.

Tseng, M. (1970). Locus of control as a determinant of job proficiency, employability, and training satisfaction of vocational rehabilitation of clients. Journal of Counseling Psychology, 17, 487-491.

Twenge, K. \& Cambpbell, W. (2002). Self-esteem and socioeconomic status: A meta-analytic review. Personality and Social Psychology Review, 6(1), 59-71.

Van der Gaag, M. (2005). Measurement of Individual Social Capital. Amsterdam: University of Groningen and Vrije Universiteit.

Van der Poel, M. (1993). Delineating personal support networks. Social Networks, 15(1), 49-70.

Visdómine-Lozano, J. \& Luciano, C. (2006). Locus de control y autorregulación conductual: revisiones conceptual y experimental. International Journal of Clinical and Health Psychology, 6(3), 729-751.

Wallston, K. (2005). The validity of the Multidimensional Health Locus of Control Scales. Journal of Health Psychology, 10, 623631.

Woolcock, M. (2001). The place of social capital in Understanding Social and Economic Outcomes. IfSUMA: Canadian Journal of Policy Research, 2(1), 1-17.

Recibido: 10 de septiembre, 2013 Aceptado: 30 de septiembre, 2013 